\title{
Consumo de medicamentos por agentes comunitários de saúde
}

\section{Consumption of medicines by community health workers}

Recebido em: 21/12/2017

Aceito em: $18 / 03 / 2018$
Dominick Danielle Mendonça SANTOS; Thamara Corrêa BARBOSA; Ricardo Lopes ROCHA; Emerson Cotta BODEVAN; Marcos Luciano Pimenta PINHEIRO

Departamento de Farmácia, Faculdade de Ciências Biológicas e da Saúde, Universidade Federal dos Vales do Jequitinhonha e Mucuri. Campus JK, Rodovia MGT 367 - Km 583, 5000, Alto da Jacuba, CEP 39100-000. Diamantina, MG, Brasil.E-mail: marcospimenta2@gmail.com

\section{ABSTRACT}

This work investigated the consumption of medicines by the Community Health Workers (CHW), and carried out a training on the subject. $44 \mathrm{CHW}$ participated in the study. The most commonly used drugs were analgesics, antipyretics and anti-inflammatories (70.8\%). The CHW reported the following frequency of drug consumption during the last month: 12 used once, six used twice, 17 used three times or more and 18 did not use medicines. Regarding the form of consumption, 18 performed self-medication. Of those interviewed, 35 had not difficulty in acquiring prescription drugs without a prescription, six used a controlled medication, and 51 respondents had used water to take the medication. The interviewees stated that they did not consume drugs influenced by commercial advertising. Based on the data obtained, it was concluded that there is the practice of self-medication and failures in the rational use of medicines among Community Health Workers.

Keywords: community health workers; self-medication; family health, strategy; drug utilization.

\section{RESUMO}

Esse trabalho investigou o consumo de medicamentos pelos agentes comunitários de saúde (ACS), e realizou uma capacitação abordando a temática. Participaram do estudo 44 ACS. Os medicamentos mais utilizados foram os analgésicos, antitérmicos e anti-inflamatórios (70,8\%). Os ACS relataram a seguinte frequência de uso de medicamentos no último mês: 12 usaram uma vez, seis usaram duas vezes, 17 usaram três vezes ou mais e 18 não usaram medicamentos. Quanto à forma de consumo, 18 realizam a automedicação. Dos entrevistados, 35 não tiveram dificuldade em adquirir medicamentos sob prescrição sem a mesma, seis fazem uso de medicamento sob controle especial e 51 entrevistados utilizam água para tomar o medicamento. Os entrevistados afirmaram não consumirem medicamentos pela influência da propaganda comercial. Diante dos dados obtidos, conclui-se que ocorre a prática da automedicação e falhas no uso racional de medicamentos entre os agentes comunitários de saúde.

Palavras Chave: agentes comunitários de saúde; automedicação; estratégia saúde da família; uso de medicamentos. 
INTRODUÇÃO

Os medicamentos constituem um insumo essencial na moderna intervenção terapêutica, sendo empregado na cura e controle de doenças quando usados racionalmente (1); entretanto, nem sempre o consumo de medicamentos acontece de forma racional, sendo que, tanto o uso indiscriminado quanto a automedicação são considerados inadequados (2).

A polimedicação, o uso abusivo de antimicrobianos, prescrições não embasadas cientificamente e o grande arsenal terapêutico disponível para o consumo levam ao uso incorreto dos medicamentos no Brasil (3), o que pode ser agravado pelo processo da automedicação, prática comum na população adulta do país (4)

No intuito de melhorar esta situação, no ano de 2004, o Ministério da Saúde estabeleceu a Política de Educação Permanente em Saúde, por meio da Portaria no 198/GM/ MS, que tinha como objetivos modificar e aperfeiçoar as ações e serviços no âmbito da saúde, além da instrução e desenvolvimento dos trabalhadores deste setor (5).

Em 2006, com objetivo de qualificar os Agentes Comunitários de Saúde (ACS) para promover o incentivo do uso racional de medicamentos nas comunidades em que atuam, o Ministério da Saúde lançou a cartilha "O trabalho dos ACS na promoção do uso correto de medicamentos", pois estes trabalhadores exercem importante função para o bom funcionamento do serviço primário de saúde (6).

A promoção do uso racional de medicamentos é um dos objetivos da Política Nacional de Medicamentos, que tem como finalidade garantir a segurança, a eficácia e a qualidade dos medicamentos. Enfatiza o processo educativo dos usuários, consumidores e profissionais, além da formação profissional em relação às prescrições medicamentosas, automedicação, dispensação e observação dos receituários para a liberação dos medicamentos sujeitos a prescrição (7), entretanto, o consumo de medicamentos nem sempre acontece de forma apropriada. Sendo que, tanto o uso indiscriminado, quanto a automedicação são considerados inadequados. Essas práticas se mostram perigosas visto que pode camuflar determinados sintomas das doenças, e também porque pode favorecer a manifestação de reações adversas, intoxicações e interações medicamentosas (8). Tais práticas podem inclusive, estar presentes entre os próprios agentes de saúde, justo os responsáveis pela orientação sobre o uso correto dos medicamentos.
Diante do exposto, o objetivo deste trabalho foi investigar o padrão de consumo de medicamentos pelos agentes comunitários de saúde das unidades básicas de saúde da zona urbana do município de Diamantina/MG e realizar capacitação acerca do uso racional de medicamentos.

\section{MÉTODOS}

Delineamento do estudo. Trata-se de um estudo transversal realizado nos postos de saúde da zona urbana da cidade de Diamantina e na Universidade Federal dos Vales do Jequitinhonha e Mucuri (UFVJM).

Contexto. O estudo contou com dois momentos distintos: coleta de dados no período de março a abril de 2016. A partir dos dados obtidos, foi realizada a segunda fase do estudo que ocorreu na Universidade Federal dos Vales do Jequitinhonha e Mucuri (UFVM), com a participação dos ACS, nos dias oito, 15 e 22 de março de 2017, e que constou de uma capacitação enfatizando os pontos observados na coleta de dados da primeira fase. No último momento de cada encontro, os ACS presentes foram divididos em grupos para discussão dos temas propostos e abordagem de situações cotidianas enfrentadas pelos mesmos, com a finalidade de buscar respostas e identificar as dificuldades. Nesta segunda fase, foram ministradas aulas expositivas pelos pesquisadores, aconteceram grupos de discussão, esclarecimento de dúvidas e ao final, os agentes comunitários de saúde apresentaram um teatro em que replicaram situações cotidianas sobre o uso racional de medicamentos e confeccionaram uma cartilha informativa baseada nas informações e conhecimentos adquiridos durante o desenvolvimento do estudo.

Participantes. Foram convidados a participar os agentes comunitários de saúde atuantes nas ESF localizadas da zona urbana do município de Diamantina, cidade da região do Vale do Jequitinhonha, MG, como uma população estimada em 48.000 habitantes, e inscritos no Cadastro Nacional de Estabelecimentos de Saúde e aceitaram participar da pesquisa por meio de assinatura do Termo de Consentimento Livre e Esclarecido (TCLE).

Variáveis. As variáveis do estudo foram sexo (masculino, feminino); número de moradores no domicílio (1 a 3, 4 a 6, 7 a 10), grau de instrução (ensino fundamental completo, ensino médio completo, ensino superior completo), quantas vezes consumiu o medicamento no último mês (nenhuma, uma, duas, três, mais de três), forma de consumo (automedicação, prescrição, ambos), motivo 
da automedicação (dificuldade de acesso aos serviços de saúde e comodidades, já sabe como usar o medicamento), classe do medicamento (Analgésicos/antitérmicos/ anti-inflamatórios, antibióticos, antigripais, sedativos/ ansiolíticos, anticoncepcionais, outros), houve dificuldade para adquirir medicamento sem prescrição (sim, não), uso de medicamento sob controle especial (sim, não), se houve reações adversas (sim, não), qual foi a forma de orientação (bula, farmacêutico, outro), se usa uso o medicamento antes ou depois as refeições (sim, não), qual o líquido utilizado para tomar o medicamento (água, leite), onde adquiriu o medicamento (campo aberto), se houve influência da propaganda (nenhuma, pouca, muita) e se já indicou ou utilizou medicamento indicado por alguém (sim, não).

Fontes de dados. Os dados foram coletados por meio do instrumento de coleta de dados semiestruturado, adaptado de Fontanella et al. (2013) (9), preenchido pelos participantes em papel.

Controle de vieses. Antes da aplicação, para melhor entendimento das questões, foi realizada uma breve explanação sobre o estudo e as questões a serem respondidas. Para facilitar as respostas e diminuir as variações das mesmas, o questionário constou de perguntas de múltipla escolha, em que foram destacados os principais problemas pesquisados. Para garantir o anonimato, os questionários após respondidos foram recolhidos em envelopes individuais não identificados, separadamente do TCLE.

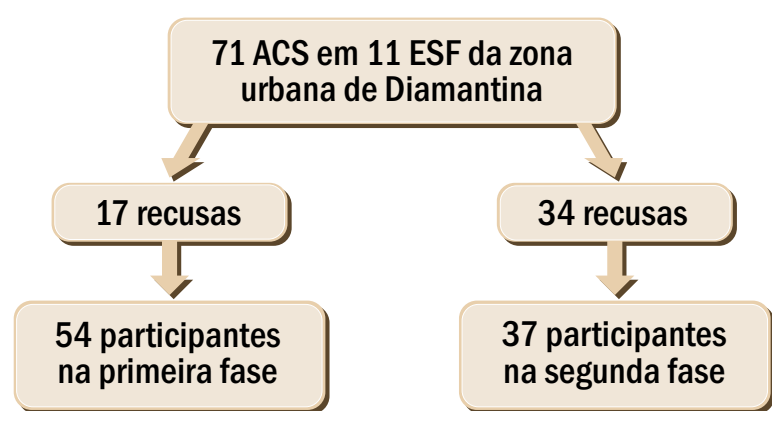

Figura 1. Processo de seleção dos participantes do estudo envolvendo agentes comunitários da Saúde (ACS), da Estratégia Saúde da Família (ESF) do município de Diamantina, MG, Brasil, no período de março a abril de 2016.

Tabela 1. Assiduidade dos agentes comunitários de saúde (ACS) de cada estratégia de saúde da família (ESF) da zona urbana de Diamantina, MG, Brasil, no período de março a abril de 2016.

\begin{tabular}{|c|c|c|c|}
\hline ESF da zona urbana & Total de ACS por ESF (n) & $\begin{array}{l}\text { Participantes na } \\
1^{\text {a }} \text { fase do estudo (n) }\end{array}$ & $\begin{array}{l}\text { Participantes na } \\
2^{\mathrm{a}} \text { fase do estudo (n) }\end{array}$ \\
\hline Arraial dos Forros & 7 & 6 & 5 \\
\hline Bom Jesus & 8 & 6 & 8 \\
\hline Bela Vista & 7 & 5 & 7 \\
\hline Cazuza & 6 & 5 & 3 \\
\hline Cidade Nova & 6 & 3 & 3 \\
\hline Gruta de Lourdes & 6 & 5 & 2 \\
\hline Jardim Imperial & 7 & 7 & 0 \\
\hline Palha & 6 & 3 & 1 \\
\hline Renascer & 6 & 5 & 4 \\
\hline Vila Operária & 6 & 5 & 4 \\
\hline Viver Melhor & 6 & 4 & 0 \\
\hline Total & 71 & $54(100.0 \%)$ & $37(100.0 \%)$ \\
\hline
\end{tabular}

Tamanho da amostra. Todos os ACS atuantes na zona urbana do município foram convidados a participar do estudo.

Métodos estatísticos. Os dados coletados foram tabulados por meio do programa Statistical Package for the Social Science (SPSS - versão 22.0), seguindo-se à análise descritiva dos mesmos.
Aspectos éticos. Este estudo recebeu aprovação pelo comitê de ética em pesquisa da Universidade Estadual de Montes Claros (Unimontes) sob o número 1.321.803/2015 e está de acordo com os princípios estabelecidos pela resolução 466/12 do Conselho Nacional de Saúde. 


\section{RESULTADOS E DISCUSSÃO}

O município de Diamantina contava com 99 ACS cadastrados no período do estudo e possuía $15 \mathrm{ESF}$, sendo 11 pertencentes à zona urbana $(\mathrm{n}=71)$ e quatro pertencentes à zona rural $(\mathrm{n}=28)$. Apesar de haver a pretensão de realizar o estudo com todos os ACS cadastrados, os ACS atuantes na zona rural não participaram do estudo devido à indisponibilidade de transporte para os pesquisadores se deslocarem até as ESF e configurou como uma das limitações deste estudo. Na primeira fase do estudo de um total de 71 ACS da zona urbana, estavam presentes 54. Na segunda fase, 37 estavam presentes (Figura 1 e Tabela 1).

Dos participantes da primeira fase do estudo, a maioria pertencia ao sexo feminino (Tabela 2), e dados semelhantes a estudos realizados em Palmas,TO, com 246 ACS em 2014 (10), no município de Lagarto, SE, com 15 ACS em 2013, e em Ribeirão Preto, SP, entre novembro de 2007 a fevereiro de 2008 com 95 agentes.

De acordo com a Lei $\mathrm{n}^{\circ} 11.350$, que regulamenta as atividades do agente comunitário de saúde, é exigido o ensino fundamental completo como escolaridade mínima (13). No presente estudo, a amostra foi composta por grande maioria que possuía este nível obrigatório de escolaridade (39 ACS, 72,2\%), diferindo dos achados de Guimarães e cols. (2017) e Marques (2008) $(10,12)$, em que predominaram o ensino médio completo; neste estudo, apenas sete ACS (13\%) possuíam ensino médio completo. Arrais e cols. (2016) em um estudo transversal de base populacional, realizado por meio dos dados da Pesquisa Nacional de Acesso, Utilização e Promoção do Uso Racional de Medicamentos coletados entre setembro de 2013 a fevereiro de 2014 (16), no qual foram entrevistados 41.433 indivíduos, observaram que os mesmos apresentavam 12 anos ou mais de estudos, diferindo também dos resultados do presente estudo, com apenas oito ACS com ensino superior completo (Tabela 2).

Quanto à habitação, a grande maioria dos pesquisados residiam com outras pessoas e em sua maioria, a habitação contava com até seis pessoas (Tabela 2). Podese afirmar que, a cada participante que admitiu fazer a automedicação, existiam mais pessoas expostas a esse risco, pois de acordo com Schwingel e cols. (2015), em um estudo transversal, realizado no município Vale do Taquari, RS (15), com 1.559 famílias, entre março a agosto de 2009, existe o costume de se estocar medicamento no domicílio. E, além disso, segundo Ascari e cols. (2014), numa pesquisa quantitativa, de delineamento transversal descritiva, realizada no município de Campos Novos, SC (16), com 200 usuários da ESF, os participantes acharam mais conveniente e cômodo consumir os medicamentos que já se encontravam no domicílio nos casos de doença ou agravo, o que pode aumentar o risco para os outros moradores em se automedicar.

Tabela 1. Dados sociodemográficos na primeira fase do estudo envolvendo agentes comunitários da Saúde (ACS), da Estratégia Saúde da Família (ESF) do município de Diamantina, MG, Brasil, no período de março a abril de 2016.

\begin{tabular}{|c|c|}
\hline Variáveis & $\mathbf{n}$ \\
\hline \multicolumn{2}{|l|}{ Sexo } \\
\hline Feminino & 50 \\
\hline Masculino & 4 \\
\hline Total & 54 \\
\hline \multicolumn{2}{|c|}{ Número de moradores no domicílio* } \\
\hline $1-3$ & 23 \\
\hline $4-6$ & 27 \\
\hline $7-10$ & 3 \\
\hline Grau de instrução & $\mathbf{n}$ \\
\hline Ensino fundamental completo & 39 \\
\hline Ensino médio completo & 7 \\
\hline Ensino superior completo & 8 \\
\hline Total & $54(100 \%)$ \\
\hline
\end{tabular}

* Houve um não-respondente

Conforme apresentado na Tabela 3, 36 participantes $(66.4 \%)$ haviam consumido algum medicamento no mês anterior à pesquisa, sendo que $34 \%$ o fizeram por meio da automedicação. Números superiores foram encontrados por Ascari e cols. (2014), com índices de 71\% (16), e por Silva e cols. (2016), em um estudo com abordagem quantitativa, realizado no município de Valparaíso, GO, de outubro a novembro de 2014 (17), com 100 clientes de uma farmácia privada, que relataram índices de $44 \%$ desta prática nos entrevistados de seus estudos. A maior parte dos entrevistados que praticaram a automedicação informou que o motivo era já saberem fazer uso dos medicamentos (Tabela 3). Guimarães e cols. (2017), em estudo descritivo,exploratório de abordagem quantitativa, realizado em Palmas, TO, com 246 ACS, entre janeiro e abril de 2014 (10), concluíram que a maioria dos ACS entrevistados afirmou não 
ter conhecimento suficiente para orientar as famílias sobre medicamentos, sentindo a necessidade de se capacitarem através de cursos específicos sobre o assunto.

No questionário aplicado, duas perguntas se referiam à automedicação. Na primeira, se o participante realizou esta prática e na segunda, o motivo para tal. Entretanto, notou-se uma incoerência, pois na primeira, apenas 18 admitiram que se automedicavam, mas na pergunta seguinte, 26 explicaram o porquê. Este fato é uma limitação do estudo, pois no momento da aplicação do questionário, o pesquisador deveria ter instruído a omitir a segunda pergunta no caso de negativa da primeira.

Entre as classes de medicamentos indicadas no questionário, $37(69,8 \%)$ relataram usar analgésicos, antitérmicos e/ou anti-inflamatórios (Tabela 3), dados semelhantes foram encontrados em pesquisa realizada em Araraquara, SP, com uma amostra de 103 participantes, em que $71,1 \%$ dos praticantes da automedicação também relataram o uso de anti-inflamatórios (18); Silva e cols. (2016), num estudo realizado no município de Valparaíso, GO, com 100 clientes de uma farmácia privada, relataram valores inferiores (44\%) nesta prática 91 (17).

Quanto à manifestação de reações adversas, foi constatado que apenas $2(3,8 \%)$ participantes relataram algum episódio, o que também foi observado no estudo de Diniz e cols. (2015), realizado com 103 participantes, obtendo dados superiores, em que $22,3 \%$ dos entrevistados relataram efeitos desagradáveis (18).

Nesta pesquisa, 35 entrevistados $(71,4 \%)$ relataram que não houve dificuldade para adquirir o medicamento desejado e todos adquiriram os medicamentos em farmácias e/ou drogarias. Oliveira e cols. (2016), em um estudo realizado em São José dos Campos, SP, em 2014, com 50 alunos do curso de Biomedicina, verificaram que $96 \%$ dos seus entrevistados responderam que conseguiram adquirir o medicamento com facilidade (19).

Apesar de todos os respondentes (53) terem adquirido os medicamentos em farmácias e/ou drogarias, apenas 11 deles $(23,9 \%)$ consultaram o farmacêutico para obter conhecimentos sobre os mesmos; $16(34,8 \%)$ consultaram a bula; e $20(43,5 \%)$ se orientaram de outra forma (Tabela 3). Um estudo transversal realizado no município de Campos Novos, SC, com 200 usuários da ESF, revelou que $16 \%$ dos entrevistados confiaram na indicação do farmacêutico.
Tabela 3. Perfil da automedicação na primeira fase do estudo envolvendo agentes comunitários da Saúde (ACS), da Estratégia Saúde da Família (ESF) do município de Diamantina, MG, Brasil, no período de março a abril de 2016.

\begin{tabular}{|c|c|}
\hline Variáveis & $\mathbf{n}$ \\
\hline \multicolumn{2}{|l|}{ Utilização de medicamento no último mês } \\
\hline Nenhuma & 18 \\
\hline Uma & 12 \\
\hline Duas & 06 \\
\hline Três & 01 \\
\hline Mais de três & 17 \\
\hline \multicolumn{2}{|l|}{ Forma de consumo ${ }^{1}$} \\
\hline Automedicação & 17 \\
\hline Prescrição & 35 \\
\hline Ambos & 01 \\
\hline \multicolumn{2}{|l|}{ Motivo da automedicação ${ }^{2}$} \\
\hline Dificuldade de acesso a serviços de saúde e comodidade & 02 \\
\hline Já sei como usar o medicamento & 24 \\
\hline \multicolumn{2}{|l|}{ Classe do medicamento ${ }^{1,3}$} \\
\hline Analgésicos/antitérmicos/anti-inflamatórios & 37 \\
\hline Antibióticos & 02 \\
\hline Antigripais & 09 \\
\hline Sedativos/ansiolíticos & 07 \\
\hline Anticoncepcionais & 15 \\
\hline Outros & 08 \\
\hline \multicolumn{2}{|c|}{ Dificuldade para adquirir medicamento sem prescrição ${ }^{4}$} \\
\hline Não & 35 \\
\hline Sim & 14 \\
\hline \multicolumn{2}{|l|}{ Medicamento controlado } \\
\hline Não & 48 \\
\hline Sim & 6 \\
\hline \multicolumn{2}{|l|}{ Reação adversa ${ }^{1}$} \\
\hline Não & 51 \\
\hline Sim & 2 \\
\hline \multicolumn{2}{|l|}{ Forma de orientação $0^{3,5}$} \\
\hline Bula & 16 \\
\hline Farmacêutico & 11 \\
\hline Outros & 20 \\
\hline \multicolumn{2}{|c|}{ Uso de medicamento antes ou depois das refeições ${ }^{4}$} \\
\hline Não & 30 \\
\hline Sim & 19 \\
\hline \multicolumn{2}{|l|}{ Líquido que usa para tomá-lo ${ }^{6}$} \\
\hline Água & 51 \\
\hline Leite & 01 \\
\hline \multicolumn{2}{|l|}{ Onde adquire o medicamento ${ }^{1}$} \\
\hline Farmácia e drogaria & 53 \\
\hline \multicolumn{2}{|l|}{ Influência da propaganda ${ }^{6}$} \\
\hline Nenhuma & 35 \\
\hline Pouca & 10 \\
\hline Muita & 07 \\
\hline \multicolumn{2}{|c|}{ Sugestão ou utilização de medicamento indicado por alguém } \\
\hline Não & 30 \\
\hline Sim & 23 \\
\hline
\end{tabular}

${ }^{1}$ um não respondeu; ${ }^{2} 28$ não responderam; ${ }^{3}$ uma mesma pessoa podia responder mais de uma opção; ${ }^{4}$ cinco não responderam; ${ }^{5}$ oito não responderam; ${ }^{6}$ dois não responderam. 
Os outros sujeitos da pesquisa $(34,8 \%)$ consultaram a bula ou outras formas de orientação (43,5\%) (16). Dados similares foram relatados por Marques (2008) (12) em um estudo transversal, realizado com 95 ACS em Ribeirão Preto, e Guimarães e cols., (2017) (10), em estudo descritivo realizado em Palmas - TO com 246 ACS em 2014, onde as bulas dos medicamentos estabeleceram a principal fonte de informação utilizada pelos ACS. Santana e cols, (2014) (11), em um estudo, transversal, realizado com 15 ACS, em 2013, discorreram quanto à importância dos ACS na identificação de erros no uso de medicamentos, pois os mesmos consideraram muito importante a orientação das famílias a respeito dos tratamentos medicamentosos, devido ao estreito contato com a comunidade, imposto pela sua profissão. Ademais, Marques (2008) (12) relata que a maior parte dos ACS entrevistados não fez curso de capacitação, e por isso consideraram não ter conhecimento suficiente para informar às famílias sobre a farmacoterapia. Nestes dois estudos, os ACS também afirmaram a necessidade de uma capacitação sobre o tema.

Quanto ao modo de uso do medicamento 51 (98,1\%) dos entrevistados utilizaram água para a ingestão dos mesmos e $19(38,8 \%)$ deles fizeram uso deles em horário próximo às refeições. Guimarães e cols, (2017) (10) observaram que os principais problemas relativos ao uso dos medicamentos estavam relacionados ao horário, dosagem, armazenamento, automedicação, validade ou medicamento diferente do prescrito. Sendo assim, percebe-se o quanto é importante orientar os usuários quanto à forma de administração, armazenamento e descarte dos medicamentos. No presente estudo, apenas dois participantes $(3,8 \%)$ relataram ter apresentado reação adversa (Tabela 3 ).

Quanto à interferência das propagandas sobre o consumo de medicamentos, 17 (32,7\%) participantes desta pesquisa assumiram alguma influência das mesmas e 25 (43,3\%) fizeram uso de algum medicamento por indicação de terceiros. No estudo de Marques (2008), 12,6\% dos ACS apontaram a televisão como influência (12).

As propagandas de medicamentos apresentadas pelas redes de televisão têm forte relação com o hábito da automedicação. Neste panorama, há uma intensa competição entre as empresas farmacêuticas, investindo crescentemente em publicidade e propaganda para seduzir consumidores, com o objetivo de aumentar as vendas e ampliar os lucros. Neste sentido, a divulgação realizada pela mídia favorece a indústria, porém não protege a sociedade dos riscos advindos da automedição (20).

$\mathrm{O}$ tema abordado na segunda fase foi o uso racional de medicamentos, na forma de um minicurso, com os seguintes conteúdos: armazenamento e descarte de medicamentos; interações medicamentosas e a automedicação e seus riscos, com carga horária total de 12 horas. Ao final, os participantes receberam uma apostila sobre a temática abordada. Esta forma de capacitação configura-se em uma estratégia simples e de baixo custo que promove o uso correto e racional de medicamentos, sendo fundamental a participação dos atores envolvidos, como por exemplo, os farmacêuticos e agentes comunitários de saúde, educando a comunidade sobre o uso dos mesmos (21). Neste estudo foi observada uma baixa participação, pois apenas 37 participantes compareceram. Este fato configura-se como uma limitação deste estudo e isso pode ser justificado por vários fatores como: o excesso de trabalho da ESF, impedindo que o os mesmos se ausentassem; dificuldade de acessibilidade, visto que algumas unidades são distantes e participação em capacitações prévias, o que poderia resultar em pouco interesse no assunto.

\section{CONCLUSÃO}

Foi observado que, em relação ao padrão de consumo dos ACS, houve falhas quanto ao uso racional de medicamentos. Por se tratar de profissionais que lidam diretamente com o público, seria desejável que tivessem maior conhecimento do assunto. Assim, é importante que as ações de educação continuada sejam constantes com os ACS, pois, lidam diariamente com os usuários das ESF, prestando informações sobre temas de saúde, incluindo o uso de medicamentos. Os resultados deste estudo poderão contribuir na busca de soluções para os problemas identificados, além de melhorar a qualidade do serviço prestado tanto para os usuários quanto para os profissionais de saúde. 
1. Leite SN, Vieira M, Veber AP. Estudos de utilização de medicamentos: uma síntese de artigos publicados no Brasil e América Latina. Ciênc. saúde coletiva. 2008;13(suppl):793-802. DOI: 10.1590/S141381232008000700029 .

2. Santos J. Consumo excessivo de medicamentos, um problema de saúde pública. Ret-SUS. 2012;(55):6-9. Disponível em: http://www.neurocienciasaudemental.xpg. com.br/art/a315.pdf

3. Wannmacher L. Condutas baseadas em evidências sobre medicamentos utilizados em atenção primária à saúde. Uso racional de medicamentos. Brasília (DF): Ministério da Saúde; 2012. p. 9-14. Disponível em: http://bvsms. saude.gov.br/bvs/publicacoes/uso_racional_medicamentos_temas_selecionados.pdf

4. Domingues PH, Galvão TF, Andrade KR, Sá PT, Silva MT, Pereira MG. Prevalence of self-medication in the adult population of Brazil: a systematic review. Rev Saude Publica. 2015;49:36. DOI: 10.1590/S00348910.2015049005709

5. BRASIL. Ministério da Saúde. Portaria no 198/GM, de 13 de fevereiro de 2004. Institui a Política Nacional de Educação Permanente em Saúde como estratégia do Sistema Único de Saúde para a formação e o desenvolvimento de trabalhadores para o setor e dá outras providências. Disponível em: www.nescon.medicina.ufmg.br/biblioteca/ imagem/1832.pdf

6. BRASIL. Ministério da Saúde. Secretaria de Ciência, Tecnologia e Insumos Estratégicos. O trabalho dos agentes comunitários de saúde na promoção do uso correto de medicamentos. Brasília: Ministério da Saúde; 2006. 2. ed. rev. Disponível em: http://bvsms.saude.gov.br/bvs/ publicacoes/trabalho_agentes_saude_promocao_medicamentos.pdf

7. BRASIL. Ministério da Saúde. Portaria $n^{\circ} 3.916$, de 30 de outubro de 1998. Institui a Política Nacional de Medicamentos e dá outras providências. Disponível em: http://bvsms.saude.gov.br/bvs/saudelegis/gm/1998/ prt3916_30_10_1998.html

8. Barroso R, Telles-Filho PCP, Pinheiro MLP, Bodevan EC, Júnior ACP; Cambraia RP. Elderly self-medication in the Family Health Strategies. Rev Enferm UFPE online. 2017;11(2):890-897. DOI: $10.5205 / \mathrm{reu}-$ ol.10263-91568-1-RV.1102sup201703

9. Fontanella FG, Galato D, Remor KVT. Perfil de automedicação em universitários dos cursos da área da saúde em uma instituição de ensino superior do sul do Brasil. Rev. Bras. Farm. 2013;94(2):154-160. 10- Guimarães MAS, Tavares NUL, Naves JOS, Sousa, MF de. Estratégia Saúde da Família e uso racional de medicamentos: o trabalho dos agentes comunitários em Palmas (TO). Trab. Educ. Saúde. 2017;15(1):183-203. DOI: 10.1590/19817746-sol00037

11. Santana VS, Lessa Júnior PM, Oliveira LAC, Silva TMR, Aguiar RG, Silveira NA, Brito GC, Carvalho AA. Avaliação das atividades e conhecimentos dos agentes comunitários de saúde do município de Lagarto-SE, quanto a promoção do uso correto de medicamentos. In: Anais do $11^{\circ}$ Congresso Internacional da Rede Unida. 2014. Fortaleza: Associação Brasileira Rede Unida; 2014. Disponível em: http://conferencias.redeunida.org. br/ocs/index.php/redeunida/RU11/paper/view/346

12. Marques TC. As atividades de agentes comunitários de saúde e a promoção do uso correto de medicamentos em unidades do Distrito de Saúde Oeste de Ribeirão Preto-SP. [Dissertação]. Ribeirão Preto: Escola de Enfermagem, Universidade de São Paulo. 2008. DOI: 10.11606/D.22.2008.tde-06102008-132626.

13. BRASIL. Lei $\mathrm{n}^{\circ} 11.350$, de 5 de outubro de 2006. Regulamenta o $\S 5^{\circ}$ do art. 198 da Constituição Federal, dispõe sobre o aproveitamento de pessoal amparado pelo parágrafo único do art. $2^{\circ}$ da Emenda Constitucional $\mathrm{n}^{\circ}$ 51 , de 14 de fevereiro de 2006, e dá outras providências. Disponível em: http://www.planalto.gov.br/ccivil_03/_ Ato2004-2006/2006/Lei/L11350.htm\#art21

14. Arrais PSD, Fernandes MEP, Pizzol TSD, Ramos LR, Mengue SS, Luiza VL, Tavares NUL, Farias MR, Oliveira MA, Bertoldi AD. Prevalência da automedicação no Brasil e fatores associados. Rev. Saúde Pública. 2016;50(supl 2):13s. DOI: 10.1590/s1518-8787.2016050006117

15. Schwingel D, Souza J de, Simonetti E, Rigo MPM, Ely LS, Castro LC, Fernandes LC, Kauffmann C. Farmácia caseira $\mathrm{x}$ uso racional de medicamentos. Cad Pedagógico, Lajeado. 2015; 12(3):117-130. DOI: 10.22410/ issn.1983-0882.v12i3a2015.973

16. Ascari RA, Ferraz L, Buss E, Rennau LR, Brum MLB. Estratégia Saúde da Família: automedicação entre os usuários. Rev. UNINGÁ Review. 2014; 18(2):42-47

17. Silva FA, Duarte HKOS, Raimundo RJS. Estudo sobre automedicação no uso de anti-inflamatórios não esteroides na cidade de Valparaíso de Goiás. Rev. Saúde e Desenvolvimento. 2016;9(5):142-153.

18. Diniz ACL, Alves GC, Furlan LC, Angelis BS de, Rodrigues B, Albaricci CB, Castro CF, Alonso JD, Marin MT, Almeida AE. A importância da promoção do uso racional de medicamentos realizada pelo Projeto de Assistência Farmacêutica Estudantil. $8^{\circ}$ Congresso de Extensão Universitária da UNESP, 2015. Disponível em: https://repositorio.unesp.br/bitstream/handle/11449/142367/ISSN2176-9761-2015-01-04-diniz. pdf? sequence $=1$ \&isAllowed $=\mathrm{y}$

19. Oliveira JAS, Fernandes WS, Ferreira RCA, Lapena SAB. Comportamento dos universitários do curso de biomedicina na realização da prática de automedicação. Rev. Bras. Educ. Saúde. 2016;6(2):05-08. DOI: 10.18378/rebes.v6i2.3444.

20. Silva JFC. Monitoramento da propaganda de medicamentos direcionada ao público leigo, veiculada em meio televisivo, frente à RDC 96/2008. [Dissertação]. Niterói: Faculdade de Farmácia, Universidade Federal Fluminense. 2015. Disponível em: http://www.repositorio.uff. br/jspui/handle/1/3112

21. Jorge JWS. Reflexões sobre o Uso Racional de Medicamentos. Pharm. Bras. 2010;78:15-16. 